\title{
Biologia reprodutiva de Tyrannus savana (Aves, Tyrannidae) em cerrado do Brasil Central
}

\author{
Miguel Ângelo Marini ${ }^{1,5}$, Yonara Lobo ${ }^{1}$, Leonardo Esteves Lopes ${ }^{2,4}$, \\ Leonardo Fernandes França ${ }^{3}$ \& Luciana Vieira de Paiva ${ }^{3}$ \\ ${ }^{1}$ Departamento de Zoologia, Instituto de Ciências Biológicas - IB, \\ Universidade de Brasília - UnB, CEP 70910-900, Brasília, DF, Brasil \\ ${ }^{2}$ Programa de Pós-graduação em Biologia Animal, Instituto de Ciências Biológicas - IB, \\ Universidade de Brasília - UnB, CEP 70910-900, Brasília, DF, Brasil \\ ${ }^{3}$ Programa de Pós-graduação em Ecologia, Instituto de Ciências Biológicas - IB, \\ Universidade de Brasília - UnB, CEP 70910-900, Brasília, DF, Brasil \\ ${ }^{4}$ Laboratório de Ornitologia, Departamento de Zoologia, Instituto de Ciências Biológicas - ICB, \\ Universidade Federal de Minas Gerais - UFMG, CEP 31270-910, Belo Horizonte, MG, Brasil \\ ${ }^{5}$ Autor para correspondência: Miguel Ângelo Marini, e-mail: marini@unb.br
}

MARINI, M.Â., LOBO, Y., LOPES, L.E., FRANÇA, L.F. \& PAIVA, L.V. 2009. Breeding biology of Tyrannus savana (Aves, Tyrannidae) in cerrado of Central Brazil. Biota Neotrop. 9(1): http://www. biotaneotropica.org.br/v9n1/en/abstract?article+bn01009012009.

\begin{abstract}
The Fork-tailed Flycatcher Tyrannus savana (Tyrannidae) is a migratory passerine that occurs in central Brazil between August and February. Its breeding biology is still little known, and this study reports data on breeding season, incubation and nestling periods, nest and egg characteristics, nest success and predation rate. We searched for nests at "Estação Ecológica de Águas Emendadas" (ESECAE) and its surroundings, Distrito Federal, Brazil, in the breeding seasons of 2002 to 2007. We monitored 78 nests each 2-4 days. Breeding season started in late September and lasted until mid December. Incubation lasted $13.6 \pm 0.4$ days $(n=21)($ mean \pm SE) and nestling period lasted $15 \pm 0.4$ days $(n=27)$. Most nests $(n=39)$ were found in "cerrado ralo" vegetation and were built on plants of the genus Kielmeyera. Clutch size ranged from 1 to 3 eggs, with an average of $2.5 \pm 0.3$ eggs. Egg length and width averaged $22.2 \pm 0.2 \mathrm{~mm}$ and $15.8 \pm 0.1 \mathrm{~mm}(\mathrm{n}=6)$, respectively, and the average weight was $3.0 \pm 0.2 \mathrm{~g}(\mathrm{n}=5)$. Thirty $(52.5 \%)$ nests were successful, $24(43.9 \%)$ were predated and only two $(3.7 \%)$ were abandoned. Egg success was $39.2 \pm 1.4 \%$ and mean hatching rate was $0.6 \pm 0.2$ fledglings/egg. Overall productivity was $1.0 \pm 0.3$ fledglings per nest, and fecundity rate was $1.4 \pm 0.4$ fledglings per female. Nest success (Mayfield method) was $45.9 \% \pm 1.1$, with no significant difference between the average daily survival rate between egg and nestling periods. Several of the estimated parameters for $T$. savana revealed to be different than expected for a tropical flycatcher.
\end{abstract}

Keywords: nesting, neotropical, predation, reproductive biology, reproductive success.

MARINI, M.Â., LOBO, Y., LOPES, L.E., FRANÇA, L.F. \& PAIVA, L.V. 2009. Biologia reprodutiva de Tyrannus savana (Aves, Tyrannidae) em cerrado do Brasil Central. Biota Neotrop. 9(1): http://www.biotaneotropica. org.br/v9n1/pt/abstract?article+bn01009012009.

Resumo: A tesourinha Tyrannus savana é um Passeriforme (Tyrannidae) migratório que ocorre no Planalto central entre os meses de agosto e fevereiro. Sua biologia reprodutiva é ainda pouco conhecida e foi abordada pelo presente estudo quanto aos seguintes aspectos: período reprodutivo, tamanho da ninhada, tempo de incubação e de permanência dos ninhegos no ninho, características dos ninhos e ovos, taxa de sucesso dos ninhos e taxa de predação. O estudo foi realizado na Estação Ecológica Águas Emendadas e em fragmentos do entorno, DF, nas estações reprodutivas de 2002 a 2007 . Os ninhos $(n=78)$ foram monitorados em intervalos de $2-4$ dias. O período reprodutivo estendeu-se de setembro a dezembro. O período médio de incubação foi de 13,6 \pm 0,4 dias $(n=21)$ (média $\pm E P)$, e a permanência dos ninhegos no ninho foi de $15 \pm 0,4$ dias $(n=27)$. A maioria dos ninhos $(n=39)$ foi encontrada em cerrado ralo e em plantas-suporte do gênero Kielmeyera. O tamanho da ninhada variou de 1 a 3 ovos com média de 2,5 $\pm 0,3$ ovos. O comprimento e a largura média dos ovos foram de 22,2 $\pm 0,2 \mathrm{~mm}$ e $15,8 \pm 0,1 \mathrm{~mm}(\mathrm{n}=6)$, respectivamente, e o peso médio foi de 3,0 $\pm 0,2 \mathrm{~g}(\mathrm{n}=5)$. Trinta ninhos $(52,5 \%)$ tiveram sucesso, $24(43,8 \%)$ foram predados e apenas dois $(3,7 \%)$ foram abandonados. O sucesso dos ovos foi de 39,2 $\pm 1,4 \%$, sendo a taxa de eclosão de 0,6 \pm 0,2 filhotes/ovo, a fecundidade de 1,5 $\pm 0,4$ filhotes/fêmea e da produção anual de filhotes de 1,0 \pm 0,3 filhotes/ninho. O sucesso dos ninhos (método de Mayfield) foi de $45,9 \% \pm 1,1$, não havendo diferença significativa entre a taxa média de sobrevivência diária nas fases de ovo e ninhego. Diversos parâmetros estimados para T. savana revelaram ser diferentes do esperado para um tiranídeo tropical.

Palavras-chave: nidificação, neotrópico, predação, biologia reprodutiva, sucesso reprodutivo. 


\section{Introdução}

A reprodução é um processo biológico fundamental na história de vida dos seres vivos, pois é por meio desta que as espécies garantem a sua sobrevivência. As atividades associadas à reprodução estão entre os comportamentos mais complexos e conspícuos das aves e muito da compreensão sobre a evolução e a função dos sistemas de acasalamento dos vertebrados provêm de estudos com aves (Pough et al. 1993). O conhecimento de vários aspectos da biologia reprodutiva de aves tropicais, tais como duração do período reprodutivo e parâmetros da história de vida ainda são escassos (Stutchbury \& Morton 2001). Tais informações são importantes não só para o desenvolvimento e investigação de hipóteses sobre aspectos evolutivos e comportamentais, mas também por serem úteis na proposição de medidas conservacionistas (Boyce 1992, Reed et al. 1998).

A família Tyrannidae engloba uma grande variedade de espécies cuja distribuição é exclusiva do continente americano e ocupa os mais variados ambientes (Fjelda \& Krabbe 1990, Ridgely \& Tudor 1994). O gênero Tyrannus, é composto por 13 espécies, seis das quais com ocorrência na América do Sul (Clements 2007). A espécie Tyrannus savana Vieillot, 1808 (tesourinha) está subdividida em quatro subespécies (Clements 2007): T. s. monachus, T. s. circumdatus, T. s. sanctaemartae e T. s. savana. O táxon nominal é o de mais ampla distribuição geográfica, encontrada desde o Uruguai, Argentina, Brasil, Bolívia, até Venezuela e Colômbia, no extremo norte da América do Sul (Zimmer 1937, Pinto 1944). Tyrannus s. savana é migratório. Reproduz-se durante a primavera e verão no centro e sul de sua área de distribuição geográfica, migrando para o norte da América do Sul, onde encontra sua área de invernada (Zimer 1937, Negret \& Negret 1981, Sick 1983, 1997, Chesser 1997). Alguns aspectos da sua biologia reprodutiva são conhecidos, como o período de incubação e de ninhego, hábitat de nidificação, estrutura do ninho e caracterização dos ovos (Novaes 1973, Fernandez 1978, Negret \& Negret 1981, Pimentel 1985, Mezquida 2002, Di Giacomo 2005). No entanto, apesar de comum e conspícua, vários parâmetros e características ainda precisam ser estudados, incluindo o período reprodutivo, tamanho da ninhada, sucesso dos ovos, taxa de eclosão, taxa de fecundidade, taxa de sobrevivência e sucesso reprodutivo. $\mathrm{O}$ objetivo do presente estudo é descrever esses aspectos da biologia reprodutiva de Tyrannus savana.

\section{Material e Métodos}

\section{1. Área de estudo}

Realizamos o estudo no bioma Cerrado, segundo maior bioma do Brasil e que ocupa $21 \%$ do território nacional (Borlaug 2002). Considerada a maior e mais ameaçada savana tropical do mundo (Silva \& Bates 2002) é um dos 'hotspots' mundiais de biodiversidade (Myers et al. 2000, Silva \& Bates 2002). A biodiversidade do Cerrado é elevada, compreendendo uma rica avifauna de mais de 850 espécies de aves (Silva 1995, Silva \& Santos 2005). O clima dessa região é estacional, apresentando um período chuvoso que dura de outubro a abril, seguido por um período seco de maio a setembro (Nimer 1979). A precipitação média anual é de 1.500 a $1.750 \mathrm{~mm}$ (Nimer 1979) e as temperaturas são geralmente amenas ao longo do ano, entre 22 e $27^{\circ} \mathrm{C}$ em média (Klink \& Machado 2005).

Realizamos a maior parte do estudo na Estação Ecológica de Águas Emendadas (ESECAE) $\left(15^{\circ} 31^{\prime}\right.$ a $15^{\circ} 36^{\prime} \mathrm{S}$ e $47^{\circ} 31^{\prime} \mathrm{a}$ $47^{\circ} 42^{\prime} \mathrm{W}, 1.000 \mathrm{~m}$ de altitude), Distrito Federal, entre 2002 e 2007. Com uma extensão de 10.500 ha, a ESECAE abriga as nascentes de afluentes das bacias Amazônica e Platina, na porção central do bioma Cerrado (Ribeiro \& Walter 1998). Coletamos dados na ESECAE, prin- cipalmente em uma área delimitada de 100 ha $(1 \times 1 \mathrm{~km})$ formando uma grade, a uma distância de aproximadamente $1,5 \mathrm{~km}$ da borda da reserva. Esta área abrange a maioria das fitofisionomias do Cerrado (Ribeiro \& Walter 1998). Dentro da área da grade, seis fitofisionomias podem ser observadas: campo limpo (7,7\% da área total), campo sujo $(5,7 \%)$, parque cerrado $(4,0 \%)$, cerrado ralo $(29,6 \%)$, cerrado típico $(51,7 \%)$ e cerrado denso $(0,3 \%)$. Trilhas abandonadas e estradas representam $1 \%$ da área de 100 ha. Também monitoramos alguns ninhos na borda da ESECAE em 2004 e em uma área fragmentada do entorno em 2006, constituída por chácaras, fazendas e fragmentos de cerrado, denominada Jardim Morumbi ( $15^{\circ} 30^{\prime}$ a $15^{\circ} 31^{\prime} \mathrm{S}$ e $47^{\circ} 37^{\prime}$ a $47^{\circ} 39^{\prime} \mathrm{W}$ ) (Borges 2008).

\section{Métodos de campo}

\subsection{Procura e monitoramento de ninhos}

A procura por ninhos no interior da ESECAE transcorreu entre os meses de setembro e dezembro dos anos de 2002 a 2007, sendo na grade e na borda da ESECAE em 2004, e na grade e nos fragmentos do entorno em 2006. Marcamos cada ninho encontrado com uma pequena fita plástica colorida colocada a uma distância mínima de $5 \mathrm{~m}$ do ninho, de modo a facilitar as checagens posteriores. Monitoramos ninhos acima de $2 \mathrm{~m}$ de altura com a ajuda de um espelho amarrado a uma haste. Monitoramos ninhos em intervalos de 2 a 4 dias e da maneira mais rápida possível a fim de minimizar qualquer interferência no comportamento dos animais e desenvolvimento dos ninhos.

\section{Análise dos dados}

Realizamos as análises abaixo apenas com ninhos de 2003 a 2007. Os ninhos de 2002, além de escassos, foram encontrados já na fase de ninhego e não tiveram sucesso sendo, portanto, desconsiderados da maioria das análises. Consideramos ninhos bem sucedidos aqueles em que pelo menos um filhote voou, tendo estes condições físicas consideradas suficientes para deixar o ninho, como rêmiges desenvolvidas e penas no corpo. Consideramos ninhos predados no caso do seu conteúdo ter desaparecido antes que os filhotes tivessem atingido um estágio de desenvolvimento tal que os permitissem deixar o ninho com sucesso. Consideramos ninhos inativos aqueles com construção completa e que permaneceram vazios e sem nenhum sinal de atividade durante sete dias de monitoramento, feitos em intervalos de 2 a 4 dias. Classificamos ninhos como abandonados quando contendo ovos por mais de 20 dias sem eclosão, embora se saiba que alguns desses ovos podem não ter eclodido por algum outro motivo, tal como falha na fertilização. Determinamos o período de incubação como sendo aquele compreendido entre a postura do último ovo e a eclosão do primeiro. Portanto, apenas ninhos encontrados em construção ou com postura incompleta, e que puderam ser monitorados até a eclosão, foram considerados para essa estimativa. Definimos o tempo de permanência dos ninhegos no ninho pelo intervalo entre a eclosão do primeiro ovo e a saída do último filhote (Robinson et al. 2000). Determinamos o período reprodutivo da espécie na região através da atividade dos ninhos. Definimos o período como sendo desde a data de início da construção do primeiro ninho até a saída do filhote do ninho mais tardio. Os valores de período de incubação e de permanência dos filhotes obtidos são estimativas da média do tempo entre duas visitas ao ninho e não representam os reais valores. Analisamos o tamanho da ninhada em cada estação reprodutiva de 2003 a 2007 contando-se o número de ovos encontrados em cada ninho, considerando apenas aqueles ninhos com a postura completa (permanência do mesmo número de ovos após duas checagens sucessivas com pelo menos dois dias de intervalo). 
Calculamos as taxas de eclosão dos ovos, de fecundidade, de produção dos filhotes e de sucesso dos ovos para os anos de 2003 a 2007. Para estas análises, foram descartados ninhos encontrados fora da grade de estudo e próximo à borda da reserva, assim como, os ninhos nos fragmentos, evitando assim ninhos de áreas com grande perturbação antropogênica. Portanto, estas taxas referem-se apenas aos ninhos do interior da reserva.

Calculamos a taxa de eclosão (Te) dos ovos para cada um dos ninhos em separado através da fórmula: $\mathrm{n}^{\circ}$ de filhotes nascidos $/ \mathrm{n}^{\circ} \mathrm{de}$ ovos da ninhada. Calculamos a média \pm EP para cada ano e a média total de todas as estações reprodutivas. Descartamos desta análise ninhos predados ainda na fase de incubação sem estabilização da quantidade de ovos, bem como ninhos já encontrados com filhotes.

Calculamos a taxa de fecundidade (Tf) anual das fêmeas para cada ano de 2003 a 2007 através da fórmula: $\mathrm{n}^{\circ}$ de filhotes eclodidos de todas as ninhadas de cada ano/ $\mathrm{n}^{\circ}$ de fêmeas presentes (Aguilar et al. 1999, Saether \& Bakke 2000). Calculamos a média \pm EP de todas as estações reprodutivas. O número de fêmeas presentes refere-se ao número de ninhos encontrados (uma fêmea por ninho).

Para o cálculo da produção anual de filhotes (Tpf) utilizamos a fórmula: $\mathrm{n}^{\circ}$ total de filhotes que deixaram o ninho em cada ano $/ \mathrm{n}^{\circ}$ total de ninhadas em cada ano (Ricklefs \& Bloom 1977). Calculamos para cada ano de 2003 a 2007 e a média \pm EP total de todas as estações reprodutivas.

Calculamos a porcentagem de sucessos dos ovos (Sc) pela seguinte razão: ( ${ }^{\circ}$ de filhotes que deixaram o ninho $\left.x 100\right) / n^{\circ}$ de ovos postos (Skutch 1966). Calculamos para cada ano de 2003 a 2007 e a média $\pm \mathrm{EP}$ total de todas as estações reprodutivas.

Calculamos o sucesso de Mayfield (1961, 1975) (Hensler \& Nichols 1981), o que representa a probabilidade de um casal gerar um ou mais filhotes após todo o ciclo do ninho. A taxa de predação aparente (número de ninhos predados/número total de ninhos) de ninhos com ovos e ninhegos também foi calculada entre 2003 e 2007.

\section{Determinação do hábitat de reprodução}

Classificamos o hábitat de construção dos ninhos encontrados entre 2002 e 2007 de acordo com a fitofisionomia em que estes se encontravam. Para determinar o hábitat de preferência para a reprodução por T. savana, calculamos a porcentagem de ninhos encontrados em cada fitofisionomia da área da grade.

\section{Caracterização dos ninhos e ovos}

Identificamos as espécies de planta-suporte dos ninhos encontrados entre 2002 e 2007 através da comparação de amostras das mesmas com exsicatas depositadas no Herbário do Instituto de Biologia da Universidade de Brasília. Medimos a altura dos ninhos em relação ao nível do solo $(\mathrm{cm})$ com o uso de uma trena, e pesamos e medimos (comprimento e largura) os ovos com auxílio, respectivamente, de um dinamômetro $(\mathrm{g})$ e paquímetro $(\mathrm{mm})$.

\section{Análises estatísticas}

Utilizamos o teste- $t$ de student para testar se houve diferença significativa em todos os anos entre: a) taxa média de predação de ovos e ninhegos; e b) taxa média de sobrevivência diária (TSD) nas fases de ovos e ninhegos através do protocolo de Mayfield. Utilizamos uma ANOVA de uma via para testar a diferença entre os tamanhos das ninhadas nos anos de estudo. Transformamos (arcoseno da raiz quadrada das porcentagens) as porcentagens antes das análises. Realizamos as análises utilizando-se BioStat 2.0 (Ayres \& Ayres-Junior 2000) adotando o nível de significância de 5\%.

\section{Resultados}

Foram encontrados 78 ninhos durante os seis anos do estudo (4 ninhos em 2002, 21 em 2003, 18 em 2004, 10 em 2005, 15 em 2006 e 10 em 2007). Dez destes ninhos, não apresentaram atividade após o encontro, sendo considerados inativos. O tamanho amostral das análises varia, pois foram desconsiderados alguns ninhos que não forneciam informações para determinadas análises.

\section{Período reprodutivo}

O período reprodutivo compreendeu os meses de setembro a dezembro nos seis anos estudados. A estação reprodutiva iniciou-se com o primeiro ninho ativo no dia 27 de setembro (dado da estação reprodutiva de 2003) e seguiu até o ninho mais tardio tornar-se inativo em 17 de dezembro (dado da estação reprodutiva de 2003). Cada estação reprodutiva durou em média $61 \pm 2$ dias. A duração do período de uma estação só pôde ser bem avaliada nos anos de 2003, 2005 e 2007 quando houve um maior número de dias de busca pelos ninhos. A média de todos os anos do registro de ninhos ativos e de maior número de ninhos com ovos teve seu pico na terceira dezena de outubro e o pico de ninhos com ninhegos na segunda dezena de novembro (Figura 1). O número de ninhos em cada ano foi baixo, por isso não foram analisados separadamente.

\section{Período de incubação e de ninhegos}

Para a maioria dos ninhos, os ninhegos (Figura 2) eclodiram no mesmo dia, caracterizando uma incubação sincrônica, sugerindo que a incubação se inicia na postura do último ovo. O período médio de incubação foi de 13,6 dias $( \pm 0,4 ; n=21)$ (Figura 3). O período médio de permanência dos ninhegos no ninho foi de 15 dias $( \pm 0,4 ; n=27)$ (Figura 3). Os valores máximos e mínimos são estimativas da média de duas visitas ao ninho e não representam os reais valores.

\section{Hábitat de nidificação e plantas suporte}

A determinação do hábitat de nidificação somente para os ninhos encontrados na grade indicou uma maior preferência para a construção de ninhos de $T$. savana em cerrado ralo. Dos 53 ninhos presentes somente na grade de estudo e cujos hábitats foram identificados, $73,1 \%$ $(\mathrm{n}=38)$ ocorreram no cerrado ralo, $15,4 \%(\mathrm{n}=8)$ no campo sujo, $7,7 \%(n=4)$ no cerrado típico e $3,8 \%(n=2)$ em parque cerrado.

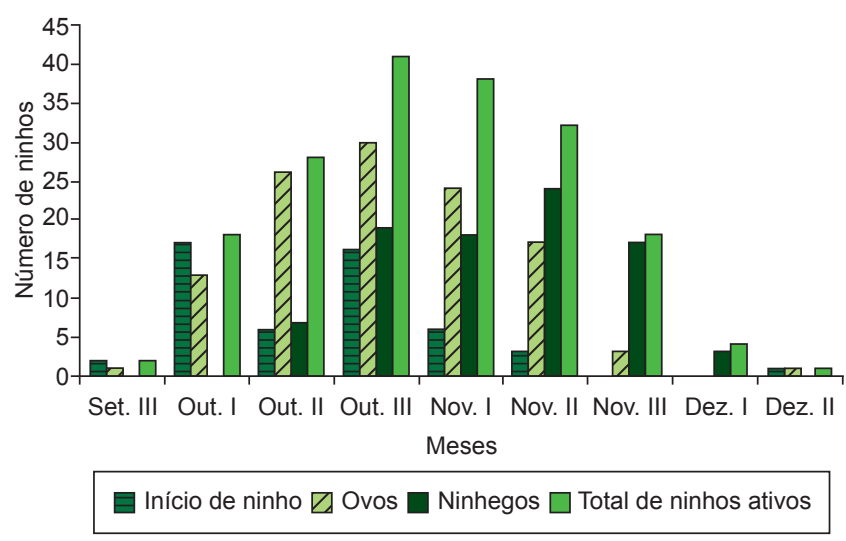

Figura 1. Número de ninhos iniciados, com ovos, com ninhegos e total de ninhos ativos de Tyrannus savana na ESECAE, Distrito Federal, em cada período de 10 dias (I, II e III) das estações reprodutivas de 2002 a 2007.

Figure 1. Number of nests initiated, with eggs, with nestlings and total active nests of Tyrannus savana in ESECAE, Distrito Federal, in each period of 10 days (I, II e III) in the breeding seasons of 2002 to 2007. 


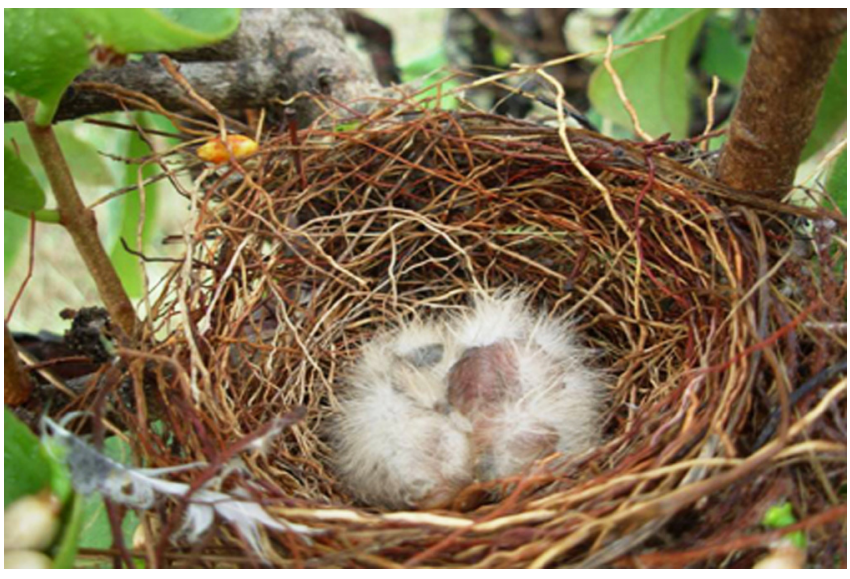

Figura 2. Filhotes de Tyrannus savana com aproximadamente três dias de desenvolvimento (Foto: Mariana B. Silveira).

Figure 2. Nestlings of Tyrannus savana with approximately three days of development (Photo: Mariana B. Silveira).

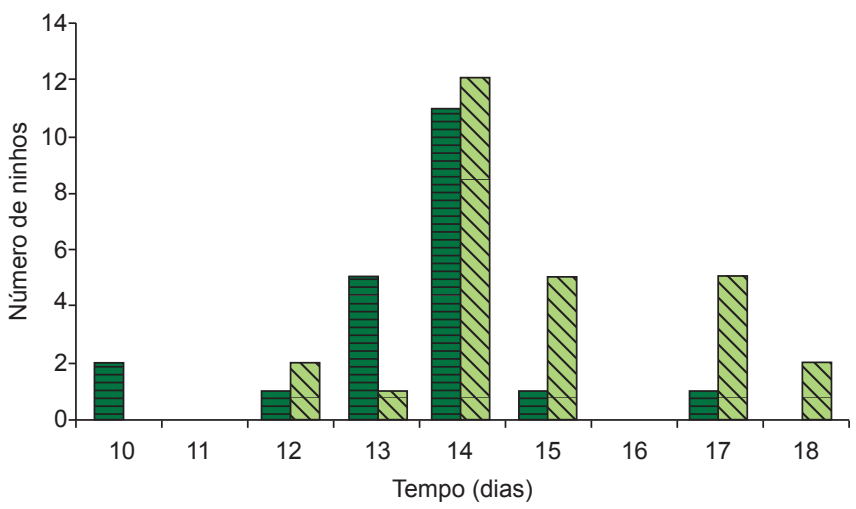

Figura 3. Tempo de incubação dos ovos (listras horizontais) e de permanência dos filhotes (listras enviesadas) de Tyrannus savana no ninho.

Figure 3. Incubation (horizontal lines) and nestling (cross-hatched lines) periods of Tyrannus savana in the nest.

Os ninhos de T. savana são normalmente abertos e em forma de tigela (Figura 2) e foram construídos, em sua maioria, sobre plantassuporte do gênero Kielmeyera Martius \& Zuccarini (Guttiferae). Dos 78 ninhos encontrados, a maioria foi construído em Kielmeyera sp., mas também em espécies da família Fabaceae, Stryphnodendron adstringens Martius e Dimorphandra mollis Schott. Entretanto, muitas das espécies de plantas-suporte não puderam ser identificadas $(43,8 \% ; n=34)$ (Figura 4). A altura média dos ninhos em relação ao nível do solo foi de 2,7 $\pm 2,0 \mathrm{~m}$, variando entre 1,2 e $6 \mathrm{~m}$.

\section{Taxas de eclosão, fecundidade, produção anual de filhotes e sucesso dos ovos}

O tamanho da ninhada variou de um a três ovos entre 2003 e 2007 , com média $2,5 \pm 0,3$ ovos $(n=52)$ e moda de três ovos. O tamanho médio da ninhada não variou significativamente $\left(\mathrm{F}_{4,47}=0,877\right.$, $\mathrm{p}=0,513$ ) entre os cinco anos de estudo (Figura 5). O comprimento e a largura média dos ovos foram $22,2 \pm 0,2 \mathrm{~mm}$ e $15,8 \pm 0,1 \mathrm{~mm}$ $(\mathrm{n}=6)$, respectivamente, e o peso médio foi de $3,0 \pm 0,2 \mathrm{~g}(\mathrm{n}=5)$.

A taxa média de sucesso dos ovos entre 2003 e 2007 foi de 39,2\% variando de 29,4 a $50 \%$ (Tabela 1). A taxa média de eclosão dos filhotes para as cinco estações reprodutivas foi de 0,6 filhotes/ovo,

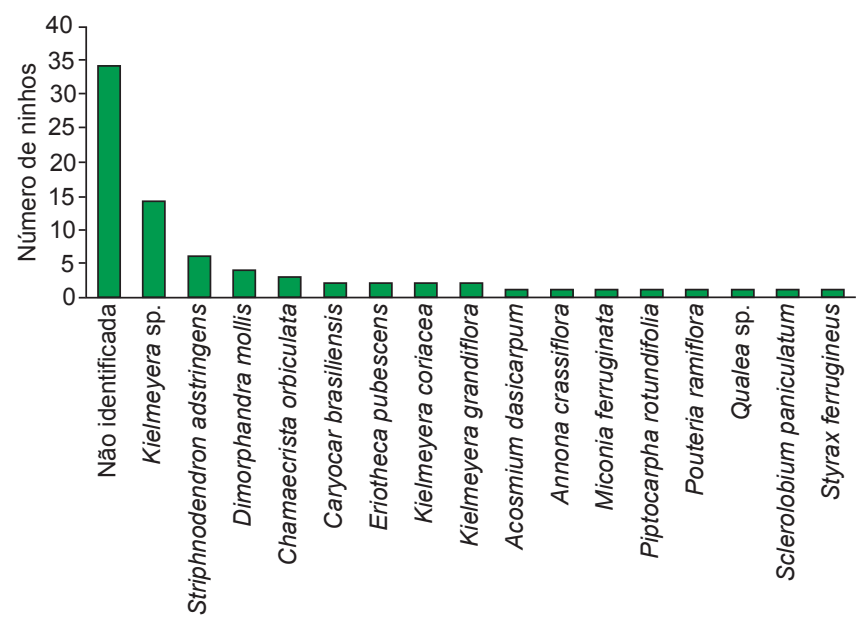

Espécies de plantas

Figura 4. Número de ninhos de Tyrannus savana construídos por espécie de planta suporte na ESECAE/DF.

Figure 4. Number of nests of Tyrannus savana built by support plant in ESECAE/DF.

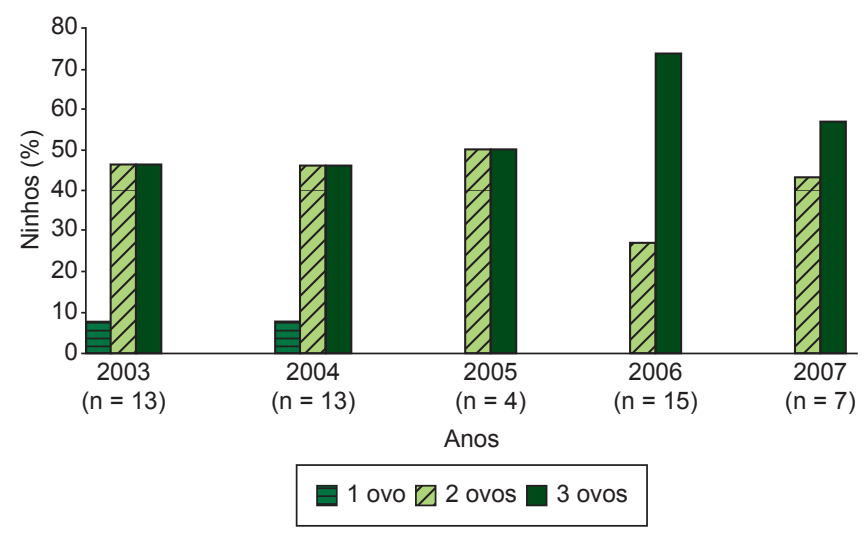

Figura 5. Porcentagem de ninhos de Tyrannus savana em relação ao tamanho da ninhada durante as estações reprodutivas de 2003 a 2007 (n = número de ninhos).

Figure 5. Percentage of Tyrannus savana nests for each clutch size during the breeding seasons from 2003 to 2007 ( $\mathrm{n}=$ sample sizes).

sendo a maior taxa registrada no ano de $2006(0,8)$ (Tabela 1). A taxa média de fecundidade foi de 1,5 filhotes/fêmea, com a maior taxa $(2,3)$ registrada em 2006 (Tabela 1). O cálculo da taxa de produção anual de filhotes teve seu maior registro também no ano de 2006, 1,3 (filhotes/ninho), e a taxa média de todos os períodos reprodutivos foi de 1,04 $\pm 0,3$ (Tabela 1 ).

\section{Destino dos ninhos}

Dos 65 ninhos monitorados entre 2003 e 2007, 56 localizavam-se dentro da grade de estudo da ESECAE. A maioria (52,5\%) dos ninhos foi bem sucedida, sendo predação a principal causa de insucesso, com poucos ninhos abandonados (Tabela 2). A taxa média de predação foi de $68 \%$ para ovos e $32 \%$ para ninhegos, a qual foi significativamente diferente entre as fases do ninho (teste $t$ com variâncias desiguais; $\mathrm{t}=2,391$, g.l $=8, \mathrm{p}=0,044)$.

A média do sucesso de Mayfield para os cinco anos foi de $45,9 \% \pm 1,1$ e variou de 35,9 a $53,5 \%$ (Figura 6). A taxa média de sobrevivência diária (TSD) nas fases de ovos e ninhegos foi de 
Tabela 1. Taxas reprodutivas (Taxa de fecundidade (Tf); Taxa de produção de filhotes (Tpf); Taxa de eclosão (Te); Sucesso dos ovos (Sc) (\%)) de Tyrannus savana de ninhos dentro da grade de estudo localizada na Estação Ecológica de Águas Emendadas durante as estações reprodutivas de 2003 a 2007.

Table 1. Reproductive rates (Fecundity (Tf); Fledgling production (Tpf); Hatching rate (Te); Egg success (Sc) (\%)) of Tyrannus savana in the studied grid in "Estação Ecológica de Águas Emendadas" during the breeding seasons of 2003 to 2007.

\begin{tabular}{ccccc}
\hline Ano & TF & TPF & Tec & Sc \\
\hline 2003 & $1,4(\mathrm{n}=13)$ & $1,00(\mathrm{n}=11)$ & $0,54(\mathrm{n}=13)$ & $41,9(\mathrm{n}=12)$ \\
2004 & $1,6(\mathrm{n}=16)$ & $1,12(\mathrm{n}=16)$ & $0,63(\mathrm{n}=15)$ & $44,4(\mathrm{n}=15)$ \\
2005 & $1,0(\mathrm{n}=8)$ & $0,55(\mathrm{n}=8)$ & $0,42(\mathrm{n}=8)$ & $29,4(\mathrm{n}=8)$ \\
2006 & $2,3(\mathrm{n}=9)$ & $1,33(\mathrm{n}=9)$ & $0,83(\mathrm{n}=9)$ & $50,0(\mathrm{n}=9)$ \\
2007 & $1,1(\mathrm{n}=8)$ & $0,75(\mathrm{n}=8)$ & $0,48(\mathrm{n}=8)$ & $30,0(\mathrm{n}=8)$ \\
Média \pm EP & $1,5 \pm 0,4(\mathrm{n}=52)$ & $1,04 \pm 0,3(\mathrm{n}=52)$ & $0,58 \pm 0,2(\mathrm{n}=53)$ & $39,2 \pm 1,4(\mathrm{n}=52)$ \\
\hline
\end{tabular}

Tabela 2. Destino dos ninhos de Tyrannus savana dentro da grade de estudo localizada na Estação Ecológica de Águas Emendadas durante as estações reprodutivas de 2003 a 2007.

Table 2. Fate of the nests of Tyrannus savana in the studied grid in "Estação Ecológica de Águas Emendadas" during the breeding seasons of 2003 to 2007.

\begin{tabular}{lrrcc}
\hline \multirow{2}{*}{ Ano } & n & Sucesso & \multicolumn{2}{c}{ Número de ninhos (\%) } \\
\cline { 3 - 5 } & & & Predado & Abandonado \\
\hline 2003 & 14 & $7(50,0)$ & $6(42,9)$ & $1(7,1)$ \\
2004 & 16 & $10(62,5)$ & $6(37,5)$ & 0 \\
2005 & 8 & $4(50,0)$ & $4(50,0)$ & 0 \\
2006 & 9 & $5(55,6)$ & $4(44,4)$ & 0 \\
2007 & 9 & $4(44,4)$ & $4(44,4)$ & $1(11,1)$ \\
Média \pm EP & 56 & $52,5 \pm 0,9$ & $43,8 \pm 0,7$ & $3,7 \pm 1,9$ \\
\hline
\end{tabular}

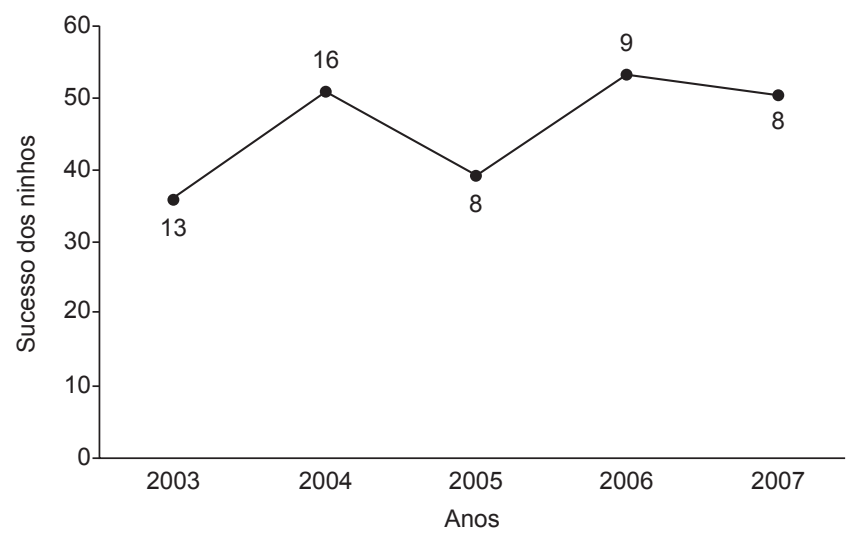

Figura 6. Sucesso de Mayfield de ninhos de Tyrannus savana variando de 35,9 a $53 \%$. Números ao lado dos pontos na figura indicam o número de ninhos amostrados.

Figure 6. Mayfield nest success of Tyrannus savana ranging from 35.9 to $53 \%$. Numbers by the dots inside the figures indicate sample sizes.

$0,96 \pm 0,02$ e $0,98 \pm 0,02$, respectivamente, não havendo diferença significativa entre elas $(t=1,552, g .1=4, p=0,195)$.

\section{Discussão}

\section{Determinação do período reprodutivo}

Os indivíduos de T. savana iniciaram sua reprodução, em média, após as primeiras chuvas de agosto/setembro, mesma época registrada para a espécie em outro estudo conduzido no Distrito Federal (INMET 2008, Pimentel 1985). Este período assemelha-se ao encontrado para várias espécies de tiranídeos (Oniki \& Willis 1983, Cavalcanti \& Pimentel 1988, Aguilar et al. 1999, Aguilar \& Marini 2007) e de Passeriformes da região centro-sul do país (Alves \& Cavalcanti 1990, Marini 1992, Duca \& Marini 2004, Pinho et al. 2006). Várias outras espécies também nidificam no mesmo período na área de estudo, incluindo os Tyrannidae Suiriri islerorum Zimmer et al., 2001 (Lopes \& Marini 2005a), Elaenia chiriquensis Lawrence, 1865 (Medeiros \& Marini 2007) e Elaenia cristata Pelzeln, 1868 (Marini et al., 2009) e os Thraupidae Neothraupis fasciata Lichtenstein, 1823 (Duca 2007) e Cypsnagra hirundinacea Lesson, 1831 (Santos 2008).

\section{Determinação do período de incubação e de ninhegos}

O período de incubação (13,6 dias) de $T$. savana na área de estudo foi menor do que encontrado em outro estudo no DF (16 dias) (Pimentel 1985) e ao descrito por Negret \& Negret (1981) (16 dias), mas semelhante ao do noroeste da Argentina (14 dias) (Mezquida 2002). O tempo de incubação foi semelhante ao de Elaenia chiriquensis (13,4 dias) (Medeiros \& Marini 2007), Neothraupis fasciata (13 dias) (Duca 2007), S. affinis Burmeister, 1856 e S. islerorum (ambos 15 dias) (Lopes \& Marini 2005a) e menor que Cypsnagra hirundinacea (16 dias) (Santos 2008), todos passeriformes encontrados na mesma área de estudo. O período médio de permanência dos ninhegos no ninho (15 dias) foi similar ao encontrado por Mezquida (2002), 14,7 dias para a mesma espécie, e idêntico ao encontrado por Medeiros \& Marini (2007) para E. chiriquensis na mesma área. Entretanto, esses valores foram bem distintos ao descrito (25 dias) por Negret \& Negret (1981) para a mesma espécie. Outros estudos encontraram valores bem inferiores para $N$. fasciata (15 a 17 e 11,7 dias) (Alves \& Cavalcanti 1990 e Duca 2007) e bem superiores para Suiriri affinis $(18,9)$ e $S$. islerorum $(18,3)$ (Lopes \& Marini 2005a). Portanto, o período de incubação registrado por nós está dentro do esperado para um tiranídeo insetívoro tropical. Períodos curtos de incubação e crescimento de filhotes são benéficos por diminuir o tempo de exposição do ninho à predação em ambos os períodos (Martin 1987). No entanto, sair cedo demais do ninho também pode expor os filhotes à predação, uma vez que o curto período de tempo no ninho pode não proporcionar um desenvolvimento suficiente para o vôo e o conseqüente escape dos predadores. Sendo assim, cada espécie pode adotar uma estratégia diferente para aumentar seu sucesso reprodutivo.

\section{Hábitat de nidificação e plantas suporte}

A preferência de $T$. savana em nidificar em ambientes com uma baixa densidade de vegetação arbórea, também foi constatada em outros estudos (Wetmore 1939, McNeil \& Itriago 1968, Novaes 1973, De Schauensee \& Phelps 1978). A espécie também pode ser observada reproduzindo em outras fitofisionomias de cerrado, bem como em áreas alteradas (Fernandez 1978, Negret et al. 1984, Pimentel 1985). 
Uma comparação do nosso estudo com o de Pimentel (1985) sugere que a densidade de ninhos de T. savana é aparentemente menor em hábitats de cerrado natural do que em hábitats alterados de Cerrado. Isto pode estar relacionado a uma menor densidade arbórea do cerrado alterado.

Tyrannus savana aparentemente seleciona plantas comuns e abundantes para construir seus ninhos. A maior utilização de espécies do gênero Kielmeyera como planta-suporte pode ter se dado em função da sua maior disponibilidade no ambiente, uma vez que elas possuem um alto índice de valor de importância (IVI) em áreas de cerrado (Silva et al. 2002). Além disso, a maioria das espécies vegetais utilizadas como suporte para os ninhos de $T$. savana são comuns em cerrado típico na ESECAE, conforme levantamento fitossociológico (Silva Jr. \& Felfili 1996).

Os ninhos de T. savana são simples em forma de tigela, construídos com raízes, gramíneas e gravetos como descritos por Negret \& Negret (1981), Pimentel (1985) e Di Giacomo (2005). Sua altura média em relação ao nível do solo $(2,7 \mathrm{~m})$ foi um pouco menor do que a encontrada para outros estudos com $T$. savana que apresentaram uma altura do ninho maior que $3 \mathrm{~m}$ (Pimentel 1985, Mezquida 2002) e maior que para outros tiranídeos encontrados na área, como E. chiriquensis (1,6 m) (Medeiros \& Marini 2007), E. cristata (1,5 m) (Marini et al. 2009) e S. islerorum (1,4 m) (Lopes \& Marini 2005a). Aparentemente existe uma ocupação diferenciada dos locais de nidificação entre as espécies de tiranídeos da área de estudo, em geral com apenas T. savana posicionando seus ninhos na extremidade de galhos altos.

\section{Taxas de eclosão, fecundidade e sucesso dos ovos}

O tamanho da ninhada de T. savana variou de um a três ovos e teve moda de três ovos, semelhante a outros estudos no DF (Negret \& Negret 1981, Pimentel 1985) e na Argentina (Mezquida 2002). Entretanto, para Euler (1900) e Salvador (1983), a postura de T. savana é de quatro a cinco ovos. Segundo Skutch (1985), tiranídeos apresentam um tamanho de ninhada bastante uniforme, de dois ovos, com exceção de poucas espécies. Uma hipótese para se explicar estas exceções é de que Passeriformes da América do Sul migratórios (como é o caso de T. savana) colocam um maior número de ovos por estação reprodutiva que as residentes (Yom-Tov et al. 1994), mas esta hipótese ainda não foi confirmada. Contrário a esta hipótese é o fato de outro tiranídeo migratório comum na área de estudo, E. chiriquensis, ter tamanho médio de ninhada de dois ovos (Medeiros \& Marini 2007).

Neste estudo, assim como, em Mezquida (2002), T. savana apresentou um ritmo de postura diário, como esperado para tiranídeos. Entretanto, segundo Skutch (1960), a maioria das espécies de tiranídeos possui um ritmo de postura a cada dois dias.

A coloração dos ovos é branca com manchas marrom-avermelhadas como descrito por Negret \& Negret (1981), sendo mais abundantes no pólo maior formando uma coroa (Di Giacomo 2005). Seu comprimento e largura média, assim como seu peso médio foram semelhantes ao encontrado por Mezquida (2002) (21,7 e 16,2 mm; $3,1 \mathrm{~g}$ ) e Di Giacomo (2005) (22 e 15,8 mm; 2,6 g).

A taxa de sucesso dos ovos foi de $39,2 \%$, superior ao encontrado na mesma área para E. cristata (31,4\%) (Marini et al. 2009) e E. chiriquensis (28\%) (Medeiros \& Marini 2007). Também existe diferença entre as taxas de sucesso de ovos em Passeriformes de regiões tropicais e temperadas, sendo esta última maior (Ricklefs \& Bloom 1977). A taxa de eclosão de ovos de T. savana de 0,6 filhotes/ ovo foi menor do que a encontrada para E. cristata $(0,95)$ (Marini et al. 2009) e E. chiriquensis (1,0) (Medeiros \& Marini 2007) na mesma área de estudo e maior que a encontrada $(0,35)$ por Skutch (1966) para aves da Costa Rica. Esta perda de aproximadamente 50\% dos ovos é semelhante ao encontrado para Leptopogon amaurocephalus
Tschudi, 1846 (Tyrannidae) (Aguilar 2001). Esta baixa taxa de eclosão pode ser devida à predação de ninhos na fase de incubação ou infertilidade dos ovos, mas não devido ao abandono dos ninhos, o qual foi raro.

A taxa de fecundidade anual representa a capacidade fisiológica e individual de cada fêmea da espécie em deixar descendentes (Saether \& Bakke 2000). A taxa de fecundidade encontrada para T. savana (1,5 filhotes/fêmea) foi superior à média encontrada para E. chiriquensis $(1,1)$ (Medeiros \& Marini 2007) e E. cristata $(0,9)$ (Marini et al. 2009), e muito maior que a de $N$. fasciata (0,31) (Duca, 2007) na mesma área. Este valor calculado deve ser próximo do valor real, pois a possibilidade das fêmeas realizarem mais de um evento reprodutivo dentro da mesma estação reprodutiva é pequena, ainda mais considerando uma estação reprodutiva tão curta como a de T. savana. A fecundidade anual está fortemente relacionada ao tamanho da ninhada e é diretamente proporcional à taxa de mortalidade do adulto (Ricklefs 2000). Portanto, o valor reprodutivo de cada ano de reprodução é mais alto para espécies com alta fecundidade, como $T$. savana. O comportamento migratório deve ocasionar maior mortalidade dos adultos de espécies migrantes do que de espécies residentes, o que pode explicar em parte as maiores taxas de fecundidade T. savana, assim como de E. chiriquensis (Medeiros \& Marini 2007), em relação às outras espécies da área de estudo.

\section{Destino e taxa de sobrevivência dos ninhos}

A porcentagem média de ninhos bem sucedidos foi de 52,5\%. Este sucesso é alto quando comparado a outros tiranídeos na mesma área de estudo, Elaenia chiriquensis (33\%) (Medeiros \& Marini 2007), Suiriri affinis (19\%) e Suiriri islerorum (14\%) (Lopes \& Marini 2005b), E. cristata (27,1\%) (Marini et al. 2009) e aos traupídeos Neothraupis fasciata (28,6\%) (Duca 2007) e Cypsnagra hirundinacea $(32,7 \%)$ (Santos 2008), e é superior à média compilada por Lopes \& Marini (2005b) para várias espécies de Tyrannidae neotropicais $(43,6 \%)$. Passeriformes em outras regiões do centro-oeste do Brasil também apresentam baixas taxas de sucesso reprodutivo (Alves 1990, Amaral \& Macedo 2003, Pinho et al. 2006). Estudos realizados com tiranídeos no estado de Minas Gerais, Brasil, apresentaram um sucesso reprodutivo de 24,4\% para Mionectes rufiventris Cabanis, 1846 (Aguilar et al. 2000), 33,8\% para Leptopogon amaurocephalus (Aguilar 2001) e 58\% para Lathrotricus euleri Cabanis, 1868 (Aguilar et al. 1999). Para alguns tiranídeos com ninhos abertos, em um estudo de 40 espécies de aves no Equador a taxa de sucesso reprodutivo variou entre $39 \%$ em Muscigralla brevicauda Orbigny \& Lafresnaye, 1837 a 59\% para Euscarthmus meloryphus Wied-Neuwied, 1831 (Marchant 1960). Estudos sobre o sucesso reprodutivo demonstram que ocorre esta ampla variação nas taxas de aves tropicais (Martin 1996). Comparativamente, as taxas de sucesso reprodutivo para aves temperadas são superiores, em torno de 50 a 80\% (Ricklefs 1969, Martin 1993). O sucesso de T. savana, portanto, é mais elevado que o esperado para um tiranídeo tropical. O seu relativo alto grau de agressividade contra predadores que se aproximam do ninho pode ser um dos fatores que ajudam a aumentar seu sucesso, o que poderia estar relacionado a uma menor sobrevivência e relativo maior investimento em cada evento reprodutivo.

A média de sucesso de Mayfield obtida (45,9\%) foi ligeiramente maior do que a encontrada para a mesma espécie em três anos de estudo, que variou de 36,9 a 41,1\% (Pimentel 1985). Esta diferença pode ser devida ao maior grau de conservação da nossa área de estudo em relação à área de Pimentel (1985). O sucesso encontrado também foi maior que o de outros dois tiranídeos, S. affinis (19\%) e S. islerorum (14\%) (Lopes \& Marini 2005b), e que o de dois traupídeos, N. fasciata (22,2\%) (Duca 2007) e C. hirundinacea (26,7\%) (Santos 2008), todos acompanhados na mesma área de estudos. Já nas florestas tropicais do 
Panamá, Robinson et al. (2000) estimaram valores de sucesso maiores, mas bastante distintos entre três espécies de Tyrannidae estudadas: Platyrinchus coronatus Sclater, 1858 (30,3\%), Mionectes oleaginea Lichtenstein, 1823 (48,9\%) e Oncostoma olivaceum Lawrence, 1862 $(71,6 \%)$. Os cálculos do sucesso pelo método de Mayfield corroboram os de predação aparente e também indicam que a espécie possui sucesso acima da média das espécies tropicais.

A defesa ativa do ninho através de comportamentos de tumulto pode ser uma das explicações para o alto sucesso reprodutivo de T. savana. Os tiranídeos freqüentemente defendem seus ninhos conspícuos de predadores (Murphy 1983). A tesourinha é reconhecidamente uma agressiva defensora de seu ninho, atacando qualquer ave que sobrevoe seu território (Sick 1997), com relatos de agressões a Falco femoralis Temminck, 1822 e F. sparverius Linnaeus, 1758 no DF (Couto 1985). No entanto, Motta-Júnior (2007) relata a predação de um adulto de T. savana que exibia comportamento de tumulto por um caburé Glaucidium brasilianum Gmelin, 1788.

O presente estudo não apresentou diferenças significativas entre as taxas médias de sobrevivência diária nas fases de ovos e ninhegos, o mesmo encontrado para outras espécies (Duca 2007, Medeiros \& Marini 2007). Entretanto, foram observadas diferenças entre as fases do ninho quando consideradas as porcentagens aparentes, diferenças estas que podem ser devidas à fase de encontro dos ninhos que pode afetar os cálculos de sobrevivência aparente. É comum se observar diferenças nas taxas de sobrevivência para fases distintas do ninho (Davis et al. 1963, Sakai 1988, Caccamise 1976, Borges 2008, Gressler 2008). Segundo a hipótese de Skutch (1985), o sucesso reprodutivo declina ao longo da estação, isso por que a predação aumentaria no período de alimentação dos filhotes, uma vez que a movimentação dos adultos ao redor do ninho para suprir os filhotes atrairia a atenção de predadores. A semelhança na sobrevivência diária entre as fases pode ser devido ao alto grau de investimento na reprodução por T. savana desde a fase de ovos.

\section{Causas da perda}

A predação foi a principal causa de perda de ninhada (43,8\%). Resultado semelhante foi encontrado para a mesma espécie em áreas alteradas, tanto na fase de incubação (59\%), como na fase de ninhegos (41\%) (Pimentel 1985). A predação tem sido enfatizada como uma importante força seletiva no processo reprodutivo das aves (Mezquida \& Marone 2001) sendo o principal fator de perda de ninhos de muitas espécies, em diferentes hábitats e localizações geográficas (Best \& Stauffer 1980, Martin 1993, Mermoz \& Reboreda 1998, Aguilar et al. 1999, 2000, Mezquida \& Marone 2002, Picman et al. 2002, Lopes \& Marini 2005b, Roper 2005, Pinho et al. 2006, Duca 2007, Medeiros \& Marini 2007). A taxa de predação de ninhos nos trópicos é alta, em torno de 80 a 90\%, mas pode variar entre regiões (Ricklefs 1969, Oniki 1979, Stutchbury \& Morton 2001, Roper 2003), sendo em geral maior que a encontrada para esta espécie. Apesar da taxa de predação de ninhos de $T$. savana ter sido menor que a esperada para uma ave tropical, ainda representa a maior causa de mortalidade em relação a outros fatores, como clima e parasitismo.

\section{Conclusão}

Diversos parâmetros reprodutivos estimados para T. savana no presente estudo revelaram ser diferentes do esperado para um tiranídeo tropical. O período reprodutivo foi mais curto que o esperado, o tamanho da ninhada foi maior, o sucesso dos ovos, a taxa de eclosão e a taxa de fecundidade também foram maiores que o esperado. Todos estes valores levaram a uma taxa de sobrevivência de ninhos e sucesso reprodutivo relativamente elevados, acima do esperado para uma espécie de passeriforme tropical. Como esperado, entretanto, o principal fator que afetou o sucesso dos ninhos foi a predação de ovos e ninhegos. A explicação para este conjunto de características pode estar relacionada ao comportamento migratório da espécie, que deve ocasionar uma alta mortalidade dos adultos, diminuindo a longevidade e consequientemente, o número de estações reprodutivas. Assim, os resultados do presente estudo indicam para $T$. savana parâmetros reprodutivos intermediários entre uma típica espécie tropical e uma espécie de região temperada.

\section{Agradecimentos}

Ao Conselho Nacional de Desenvolvimento Científico e Tecnológico (CNPq), Fundação de Apoio à Pesquisa do DF (FAP-DF), O Fundo de Pesquisa (FUNPE) e Universidade de Brasília pelos auxílios financeiros. Ao $\mathrm{CNPq}$ pela bolsa de produtividade em pesquisa a MÂM e ao CNPq e à Coordenação de Aperfeiçoamento de Pessoal de Nível Superior (CAPES) pelas bolsas de estudo a LEL, LF e LVP. Aos administradores da Estação Ecológica de Águas Emendadas (ESECAE)/Secretaria de Estado de Desenvolvimento Urbano e Meio Ambiente (SEMARH) pela autorização de estudos. Aos companheiros de campo. Aos revisores anônimos pelos comentários.

\section{Referências Bibliográficas}

AGUILAR, T.M. 2001. Biologia reprodutiva e seleção de hábitat por Leptopogon amaurocephalus (Aves: Tyrannidae), em fragmentos de mata atlântica em Minas Gerais. Dissertação de Mestrado, Universidade Federal de Minas Gerais, Belo Horizonte.

AGUILAR, T.M. \& MARINI, M.Â. 2007. Nest and nest-site reuse within and between breeding seasons by three neotropical flycatchers (Tyrannidae). Braz. J. Biol. 67(3):537-540.

AGUILAR, T.M., LEITE, L.O. \& MARINI, M.A. 1999. Biologia de nidificação de Lathrotricus euleri (Cabanis 1968) [sic] (Tyrannidae) em fragmento de mata em Minas Gerais. Ararajuba, 7(2):125-133.

AGUILAR, T.M., MALDONADO-COELHO, M. \& MARINI, M.A. 2000. Nesting biology of the Gray-hooded Flycatcher (Mionectes rufiventris). Ornitol. Neotrop. 11(3):223-230.

ALVES, M.A.S. \& CAVALCANTI, R.B. 1990. Ninhos, ovos e crescimento de filhotes de Neothraupis fasciata. Ararajuba, 1:91-94.

ALVES, M.A.S. 1990. Social system and helping behavior in the white-banded tanager (Neothraupis fasciata). Condor, 92(2):470-474.

AMARAL, M.F. \& MACEDO, R.H.F. 2003. Breeding patterns and habitat use in the endemic Curl-crested Jay of central Brazil. J. Field Ornithol. 74(4):331-340.

AYRES, M. \& AYRES Jr., M. 2000. BioStat 2.0: aplicações estatísticas nas áreas das ciências biológicas e médicas. Sociedade Civil Mamirauá; CNPq, Belém; Brasília.

BEST, L.B. \& STAUFFER, D.F. 1980. Factors affecting nesting success in riparian bird communities. Condor, 82(2):149-158.

BORGES, F.J.A. 2008. Efeitos da fragmentação sobre o sucesso reprodutivo de aves em uma região de Cerrado no Distrito Federal. Dissertação de Mestrado, Universidade de Brasília, Brasília.

BORLAUG, N.E. 2002. Feeding a world of 10 billion people: the miracle ahead. In Global warming and other eco-myths (R. Bailey, ed.). Competitive Enterprise Institute, Roseville, p.221-228.

BOYCE, M.S. 1992. Population viability analysis. Ann. Rev. Ecol. Syst. 23:481-506.

CACCAMISE, D.F. 1976. Nesting mortality in the Red-winged Blackbird. Auk, 93(3):517-534.

CAVALCANTI, R.B. \& PIMENTEL, T.M. 1988. Shiny Cowbird parasitism in central Brazil. Condor, 90(1):40-43.

CHESSER, R.T. 1997. Patterns of seasonal and geographical distribution of austral migrant flycatchers (Tyrannidae) in Bolivia. Ornithol. Monogr. 48:171-204.

CLEMENTS, J.F. 2007. The clements checklist of the birds of the World. Cornell University Press, New York. 
COUTO, E.A. 1985. O efeito da sazonalidade na população da rolinha (Columbia talpacoti) no DF. Dissertação de Mestrado, Universidade de Brasília, Brasília.

DAVIS, J., FISHER, G.F. \& DAVIS, B.S. 1963. The breeding of the Western Flycatcher. Condor, 65(5):337-382.

De SCHAUENSEE, R.M. \& PHELPS, W.H. 1978. A guide to the birds of Venezuela. Princeton University Press, Princeton.

Di GIACOMO, A.G. 2005. Aves de la Reserva El Bagual. In Historia natural y paisaje de la reserva el bagual, provincia de Formosa (A.G. Di Giacomo $\&$ S.F. RAPOVICKAS, eds.). Asociación Ornitológica del Plata, Buenos Aires, p. 201-465.

DUCA, C.G.D. 2007. Biologia e conservação de Neothraupis fasciata (Aves: Thraupidae) no cerrado do Brasil Central. Tese de Doutorado, Universidade de Brasília, Brasília.

DUCA, C. \& MARINI, M.Â. 2004. Aspectos da nidificação de Cacicus haemorrhous (Passeriformes, Icterinae) no sudeste do Brasil. Ararajuba, 12(1):25-32.

EULER, C. 1900. Descrição dos ninhos de aves do Brasil. Rev. Mus. Paul. 4:9-148.

FERNANDEZ, R.A.N. 1978. O comportamento alimentar de isolamento ecológico em oito espécies de Tyrannidae (Aves) do Planalto Central, Brasil. Dissertação de Mestrado, Universidade de Brasília, Brasília.

FJELDA, J. \& KRABBE, N. 1990. Birds of the high Andes. Apollo Books; University of Copenhagen, Copenhagen.

GRESSLER, D.T. 2008. Biologia e sucesso reprodutivo de Sicalis citrina Pelzeln 1870 (Aves: Emberizidae) no Distrito Federal. Dissertação de Mestrado, Universidade de Brasília, Brasília.

HENSLER, G.L. \& NICHOLS, J.D. 1981. The mayfield methods of estimating nesting success: a model, estimators and simulation result. Wilson Bull. 93(1):42-53.

Instituto Nacional de Meteorologia - INMET. 2008. Parâmetros meteorológicos do Distrito Federal. Ministério da Agricultura e Pecuária; Instituto Nacional de Meteorologia, Brasília. Disponível em: http://www.inmet. gov.br. (último acesso em 20/02/2008)

KLINK, C.A. \& MACHADO, R.B. 2005. A conservação do cerrado brasileiro. Megadiversidade, 1(1):147-155.

LOPES, L.E. \& MARINI, M.A. 2005a. Biologia reprodutiva de Suiriri affinis e $S$. islerorum (Aves: Tyrannidae) no Cerrado do Brasil Central. Pap. Avul. Zool. 45(12):127-141.

LOPES, L.E. \& MARINI, M.A. 2005b. Low reproductive success of Campo Suiriri (Suiriri affinis) and Chapada Flycatcher (S. islerorum) in the central Brazilian Cerrado. Bird Conserv. Int. 15(4):337-346.

MARCHANT, S. 1960. The breeding of some S.W. Ecuadorian birds. Ibis, 102:349-382.

MARINI, M.Â. 1992. Notes on the breeding and reproductive biology of the Helmeted Manakin. Wilson Bull. 104(1):169-173.

MARINI, M.Â., SILVEIRA, M.B., SOUSA, N.M. \& BORGES, F.J. 2009. Biologia reprodutiva de Elaenia cristata (Tyrannidae) no Cerrado do Brasil Central. Neotropical Biology and Conservation 4(1):3-12.

MARTIN, T.E. 1987. Food as a limit on breeding birds: a life-history perspective. Ann. Rev. Ecol. Syst. 18:453-487.

MARTIN, T.E. 1993. Nest predation among vegetation layers and habitat types: revising dogmas. Am. Nat. 141(6):897-913.

MARTIN, T.E. 1996. Life history evolution in tropical and south temperate birds: what do we really know? J. Avian Biol. 27(4):263-272.

MAYFIELD, H. 1961. Nesting success calculated from exposure. Wilson Bull. 73(3):255-261.

MAYFIELD, H. 1975. Suggestions for calculating nest success. Wilson Bull. 87(4):456-466.

McNEIL, R. \& ITRIAGO, M.C. 1968. Fat deposition in the Scissor-tailed Flycatcher (Muscivora t. tyrannus) and the Small-billed Elaenia (Elaenia parvirostris) during the austral migratory period in northern Venezuela. Can. J. Zool. 46(2):123-128.
MEDEIROS, R.C.S. \& MARINI, M.Â. 2007. Biologia reprodutiva de Elaenia chiriquensis (Lawrence) (Aves: Tyrannidae) em cerrado do Brasil Central. Rev. Bras. Zool. 24(1):12-20.

MERMOZ, M.E. \& REBOREDA, J.C. 1998. Nesting success in Brown-andyellow Marsh-birds: effects of timing, nest site, and brood parasitism. Auk, 115(4):871-878.

MEZQUIDA, E.T. \& MARONE, L. 2001. Factors affecting nesting success of a bird assembly in the central Monte Desert, Argentina. J. Avian Biol. 32(4):287-296.

MEZQUIDA, E.T. 2002. Nidificación de ocho especies de Tyrannidae en la Reserva de Ñacuñán, Mendoza, Argentina. Hornero, 17:31-40.

MEZQUIDA, E.T. \& MARONE, L. 2002. Microhabitat structure and avian nest predation risk in an open Argentinean woodland: an experimental study. Acta Oecol. 23(5):313-320.

MOTTA Jr., J.C. 2007. Ferruginous Pygmy-owl (Glaucidium brasilianum) predation on a mobbing Fork-tailed Flycatcher (Tyrannus savana) in south-east Brazil. Biota Neotrop. 7(2): http://www.biotaneotropica. org.br/v7n2/pt/abstract?short-communication+bn04407022007 (último acesso em 09/04/2008).

MURPHY, M.T. 1983. Nest success and nesting habitats of Eastern Kingbirds and other flycatchers. Condor, 85(2): 208-219.

MYERS, N., MITTERMEIER, R.A., MITTERMEIER, C.G., Da FONSECA, G.A.B. \& KENT, J. 2000. Biodiversity hotspots for conservation priorities. Nature 403(6772):853-858.

NEGRET, A., TAYLOR, J., SOARES, R.C., CAVALCANTI, R.B. \& JOHNSON, C. 1984. Aves da região geopolítica do Distrito Federal. Ministério do Interior; Secretaria Especial do Meio Ambiente, Brasília.

NEGRET, A.J. \& NEGRET, R. 1981. As aves migratórias do Distrito Federal. Instituto Brasileiro de Desenvolvimento Florestal, Brasília.

NIMER, E. 1979. Climatologia no Brasil. Instituto Brasileiro de Geografia e Estatística; Superintendência de Recursos Naturais e Meio Ambiente, Rio de Janeiro.

NOVAES, F.C. 1973. Aves de uma vegetação secundária na foz do Amazonas. Museu Paraense Emílio Goeldi, Belém.

ONIKI, Y. 1979. Is nesting success of birds low in the trop ics? Biotropica, 11:60-69.

ONIKI, Y. \& WILLIS, E.O. 1983. Breeding records of birds from Manaus, Brazil; IV. Tyrannidae to Vireonidae. Rev. Bras. Biol. 43(1):45-54.

PICMAN, J., PRIBIL, S. \& ISABELLE, A. 2002. Antipredation value of colonial nesting in Yellow-headed Blackbirds. Auk, 119(2):461-472.

PIMENTEL, T.M. 1985. Biologia reprodutiva de Tyrannus savana (Aves: Tyrannidae), com uma comparação entre o forrageamento desta espécie e de T. melanacholicus no Planalto Central. Dissertação de Mestrado, Universidade de Brasília, Brasília.

PINHO, J.B., LOPES, L.E., MORAIS, D.H. \& FERNANDES, A.M. 2006. Life history of the Mato Grosso Antbird Cercomacra melanaria in the Brazilian Pantanal. Ibis, 148(2):321-329.

PINTO, O.M. 1944. Catálago das aves do Brasil e lista dos exemplares existentes na coleção do Departamento de Zoologia - $2^{\mathrm{a}}$ parte. Publicação do Departamento de Zoologia, São Paulo.

POUGH, F.H., HEISER, J.B. \& McFARLAND, W.N. 1993. A vida dos vertebrados. 3 ed. Atheneu, São Paulo.

REED, J.M., ELPHICK, C.S. \& ORING, L.W. 1998. Life-history and viability analysis of the endangered Hawaiian Stilt. Biol. Conserv. 84(1):35-45.

RIBEIRO, J.F. \& WALTER, B.M.T. 1998. Fitofisionomias do bioma cerrado. In Cerrado: ambiente e flora (S.M. Sano \& S.P. Almeida, eds.). EMBRAPA, Planaltina, p. 89-166.

RICKLEFS, R.E. 1969. An analysis of nesting mortality in birds. Smiths. Contrib. Zool. 9:1-47.

RICKLEFS, R.E. 2000. Density dependence, evolutionary optimization, and the diversification of avian life histories. Condor, 102(1):9-22.

RICKLEFS, R.E. \& BLOOM, G. 1977. Components of avian breeding productivity. Auk, 94:86-96. 
RIDGELY, R.S. \& TUDOR, G. 1994. The birds of South America, vol. 2, the Suboscine Passerines. University of Texas Press, Austin.

ROBINSON, W.D., ROBINSON, T.R., ROBINSON, S.K. \& BRAWN, J.D 2000. Nesting success of understory forest birds in Central Panama. J. Avian Biol. 31(2):151-164.

ROPER, J.J. 2003. Nest-sites influence predation differently at natural and experimental nests. Ornitol. Neotrop. 14(1):1-14.

ROPER, J.J. 2005. Try and try again: nest predation favors persistence in a neotropical bird. Ornitol. Neotrop. 16(3):253-262.

SAETHER, B.E. \& BAKKE, O. 2000. Avian life history variation and contribution of demographic traits to the population growth rate. Ecology, 81(3):642-653.

SAKAI, H.F. 1988. Breeding biology and behavior of Hammond's and Western Flycatchers in Northwestern California. Western Birds, 19(2):49-60.

SALVADOR, S.A. 1983. Parasitismo de cria del renegrido (Molothrus bonariensis) en Villa María, Córdoba, Argentina (Aves: Icteridae). Hist. Nat. 3:149-158.

SANTOS, L.R. 2008. Biologia reprodutiva e comportamento cooperativo em ninhos de Cypsnagra hirundinacea. Dissertação de Mestrado, Universidade de Brasília, Brasília.

SICK, H. 1983. Migrações de aves na América do Sul Continental. Centro Nacional de Pesquisa para Conservação das Aves Silvestres, Brasília.

SICK, H. 1997. Ornitologia brasileira. Nova Fronteira, Rio de Janeiro.

SILVA Jr., M.C. \& FELFILI, J.M. 1996. A vegetação da estação ecológica de águas emendadas. Secretaria de Meio Ambiente, Ciência e Tecnologia do Distrito Federal, Brasília.

SILVA, J.M.C. 1995. Birds of the Cerrado region, South America. Steenstrupia 21(1):69-92.

SILVA, J.M.C. \& BATES, J.M. 2002. Biogeographic patterns and conservation in the South American Cerrado: a tropical savanna hotspot. Bioscience, 52(3):225-233.
SILVA, J.M.C. \& SANTOS, M.P.D. 2005. A importância relativa dos processos biogeográficos na formação da avifauna do Cerrado e de outros biomas brasileiros. In Cerrado: ecologia, biodiversidade e conservação (A. Scariot, J.C. Souza-Silva \& J.M. Felfili, eds.). Ministério do Meio Ambiente, Brasília, p.220-233.

SILVA, L.O., COSTA, D.A., SANTO FILHO, K.E., FERREIRA, H.D. \& BRANDÃO, D. 2002. Levantamento florístico e fitossociológico em duas áreas de Cerrado sensu strictu no Parque Estadual da Serra de Caldas Novas, Goiás. Acta Bot. Bras. 16(1):43-53.

SKUTCH, A.F. 1960. Life histories of Central American Birds. v.1. Pacific Coast Avifauna 34, Cooper Ornithological Society, Berkeley.

SKUTCH, A.F. 1966. A breeding bird census and nesting success in Central America. Ibis, 108(1):1-16.

SKUTCH, A.F. 1985. Clutch size, nesting success, and predation on nests of neotropical birds, reviewed. Ornithol. Monogr. 36:575-593.

STUTCHBURY, B.J.M. \& MORTON, E.S. 2001. Behavioral ecology of tropical birds. Academic Press, San Diego.

WETMORE, A. 1939. Observations on the birds of Northern Venezuela. Proc. U. S. Nat. Mus. 87:173-260.

YOM-TOV, Y., CHRISTIE, M.I. \& IGLESIAS, G.J. 1994. Clutch size in passerines of southern South America. Condor, 96(1):170-177.

ZIMMER, J.T. 1937. Notes on the genera Muscivora, Tyrannus, Empidonomus and Sirystes, with further notes on Knipolegus. Studies of Peruvian Birds. Am. Mus. Novit. (962):1-28.

Recebido em 25/08/08 Versão reformulada recebida em 05/01/09 Publicado em 19/01/09 
